\title{
International Symposium to Address Clusters and Nano-Assemblies in Physical and Biological Systems
}

The International Symposium on Clusters and Nano-Assemblies: Physical and Biological Systems (ISCANA) will be held in Richmond, Virginia, November 10-13, 2003. The symposium will deal with the fundamental science and technology of atomic clusters and nanostructures and their assemblies in physical and biological systems. The meeting will also investigate how finite size, low dimensionality, and reduced symmetry affect properties.

While the study of clusters and nanostructures has been actively pursued in the physical sciences for only the past two decades, nanoscale benefits in biological systems have been known for a much longer time. The focus of this symposium is to explore ways by which an understanding of the unique properties of nanoscale biological systems such as proteins, enzyme reactions, RNA, and DNA can help researchers design novel materials composed of inorganic nanoscale sys- tems in physical sciences. Similarly, the symposium will explore how the techniques developed in physical sciences can lead to a fundamental understanding of biological systems. The study of proteins in the gas phase through electrospray ionization mass spectroscopy is an example of how the interface between the physical and biological sciences can lead to major breakthroughs. The symposium is aimed at taking a step in this direction.

The topics to be covered include synthesis; nucleation; growth; characterization; atomic and electronic structure; dynamics; ultrafast spectroscopy; stability; electrical, magnetic, optical, thermodynamic, and catalytic properties of clusters; cluster materials; nanostructures; and biological systems, including supramolecular assemblies, biointerfaces, and biohybrid devices. The symposium will also address areas of nanotechnology, including spintronics; magnetic, optical, and biosensors; catalysts; molecular electronics; drug delivery; and biochips.

This is an interdisciplinary symposium drawing participants and speakers from physics, chemistry, biology, materials science, and chemical and electrical engineering. The synergism produced by bringing researchers from multidisciplinary areas is expected to take this field a step closer to realizing its potential for novel technologies.

The abstract deadline is August 1, 2003.

The symposium is endorsed by the Materials Research Society, American Physical Society, ASM International, and The Minerals, Metals \& Materials Society. For more information, contact ISCANA, c/o P. Jena, Physics Department, Virginia Commonwealth University, Richmond, Virginia 23284-2000; 804-828-8991; fax 804828-7073; e-mail iscana@vcu.edu; or access Web site www.vcu.edu/ISCANA/. 